\title{
PRODUCCIÓN DE SALUD Y MORTALIDAD
}

\author{
E. R. Weiss-AltANER \\ El Colegio de México
}

\section{INTRODUCCIÓN}

EN EL Último decenio surgió una notable revisión de la teoría subjetivista del consumo $(1 ; 5: 1322-24 ; 7 ; 8)$, la cual, al incorporar en su marco teórico el tiempo de trabajo como un insumo escaso, permití la interpretación del consumo como una actividad productiva. Desde este punto de partida - prefigurado mucho antes en el pensamiento económico (9: 10-14, 101; 11: 27) - la microeconomía neoclásica pudo abordar el análisis de varios aspectos de la vida doméstica, como fecundidad, matrimonio y la participación de la mujer en actividades productivas fuera de su hogar (véanse por ejemplo 13, 15, 16).

Según la nueva interpretación, la satisfacción subjetiva del consumidor no provendría directamente de los bienes que él ha comprado o producido, sino de un flujo de servicios de consumo que son producidos en el mismo hogar del consumidor. El hogar del consumidor combinaría su tiempo de trabajo con los bienes comprados o producidos en el mismo hogar, elaborando éstos, para así producir el flujo de servicios que sería la verdadera fuente del goce subjetivo que constituye el consumo. Por ejemplo, no serían los comestibles mismos los que proporcionarían satisfacción subjetiva de manera directa, sino que ellos, junto con otros bienes (por ejemplo, utensilios, cocina, combustible), serían transformados por el trabajo hogareño - de acuerdo con la tecnología vigente en la preparación de comestibles - en el flujo de servicios de "alimentación", el cual sî sería la fuente directa del goce personal del consumidor.

Esto significa que el consumo ocasiona al consumidor gastos mayores que el precio del bien de consumo (o mayores que su costo de producción, si no se trata de mercancías). El costo del servicio de consumo comprendería: 1) el precio de las mercancías más el costo de los otros bienes, producidos en el hogar del consumidor, que son elaborados en el hogar para producir los servicios de consumo: 2) el gasto de tiempo de trabajo del consumidor en: a) informarse sobre y conseguir las mercancías por elaborar, y b) transformar esas mercancías, junto con otros bienes que no son mercancías, para producir los servicios que, según la nueva teoría, serían los verdaderos objetos de consumo.

El propósito de este ensayo es comenzar a elaborar un esquema que serviría como instrumento de trabajo para expicar tendencias y diferen- 
ciales en mortalidad y morbilidad por hogares, destacando el hecho de que la salud no es algo que las personas reciben, sino que es algo que ellas producen. Esto último lo indica la interpretación de actividades hogareñas de consumo como actividades productivas, en las que descuella el tiempo de trabajo gastado por los miembros del hogar en actividades hogareñas. Los adjetivos "hogareño" y "extrahogareño" ubican las actividades dentro o fuera del hogar, respectivamente. Por ejemplo, el trabajo de miembros del hogar en su propiedad agrícola caería bajo el renglón de actividad hogareña, mientras que el trabajo de jornaleros contratados o forzados en esa misma propiedad sería actividad extrahogareña de los mismos. Esta distinción refleja la organización de la mayoría de las sociedades actuales en grupos de tamaño mucho menor que la población total, lo cual, por cierto, es históricamente determinado. Diferentes organizaciones sociales caben en el esquema por plantear, sin modificar lo esencial: la interpretación de la salud como un producto conjunto de muchas actividades de producción y consumo. "Hogareño" es preferido a "familiar", para poder indicar a las agrupaciones en las que no todos los miembros estén unidos por lazos de parentesco. Finalmente, en lo que sigue, los términos "actividades de producción y consumo" y "actividades" serán intercambiables.

\section{EL ESQUEMA}

La mortalidad y la morbilidad hogareñas reflejan el nivel de salud del hogar, nivel que provendría, en primera instancia, de la interacción entre 1) la demanda hogareña de salud, 2) la disponibilidad hogareña de salud, 3) la disponibilidad ambiental de salud, 4) la virulencia de los agentes patógenos y de los vectores de enfermedad, y 5) la composición genética de la población. Todas las variables son referidas al mismo período de tiempo, aunque seguramente hay relaciones de retraso que habría que especificar al utilizar el presente esquema en la investigación concreta. La demanda hogareña de salud sería la magnitud de salud deseada por un hogar para cada uno de sus miembros. El lado de la oferta o disponibilidad de salud es más complejo, porque la salud es un producto conjunto ${ }^{1}$ de una amplia gama de actividades, muchas de las cuales escapan al control del hogar.

A la salud observada de un hogar determinado contribuyen:

1) aquellas actividades hogareñas de producción y consumo, de ese hogar, en las que la buena o mala salud de los miembros del hogar es un producto conjunto;

1 En el caso de la salud se observan tanto la producción como la demanda conjuntas, ambas de origen técnico. Por ejemplo, la producción de nutrición en el hogar también brinda salud, y lo mismo sucede con otros servicios de consumo producidos en el hogar, lo que hace de la salud un producto conjunto de gran número de actividades domésticas. Otro ejemplo es la extracción de carbón, que produce enfermedades respiratorias al mismo tiempo que carbón. 
2) las externalidades, en materia de salud, de las actividades hogareñas de producción y consumo de otros hogares. Por ejemplo, la basura que arrojan las actividades hogareñas puede afectar la salud de hogares cercanos;

3) las externalidades, en materia de salud, de las actividades extrahogareñas de producción y consumo. Por ejemplo, al mismo tiempo que una fábrica produce cemento, puede estar contaminando el aire de la comunidad en la que se encuentra, dañando la salud de las personas que ahí viven;

4) los efectos de las actividades extrahogareñas sobre la salud de las personas que participan en ellas. Por ejemplo, el obrero en la fábrica de cemento recién mencionada ve su salud afectada por las condiciones de trabajo, o sea que produce cemento y parte de su salud a la vez.

Desde el punto de vista de un hogar determinado, el primero de los cuatro renglones recién mencionados es la disponibilidad hogareña de salud: el aporte de las actividades de producción y consumo, en el hogar en cuestión, a la magnitud de salud observada de ese mismo hogar. Los otros tres renglones representan los componentes de la disponibilidad ambiental de salud para ese hogar: la contribución al nivel de salud del hogar en cuestión, atribuible a) a externalidades, en materia de salud de las actividades de producción y consumo en otros hogares y en ubicaciones extrahogareñas, y $b$ ) a la participación de miembros del hogar bajo consideración en actividades fuera de su hogar. La agregación por familias, hasta llegar al nivel de toda la sociedad, preserva la distinción entre disponibilidad hogareña y disponibilidad ambiental de salud.

Los cambios en la virulencia de los agentes patógenos y de los vectores de enfermedad, así como en la composición genética de la población, afectan la salud personal con mucho retraso, y no sienten un impacto inmediato de parte de las actividades actuales de producción(3). Por consiguiente, no volveré a tratarlas en lo que sigue.

Finalmente, la interacción entre los cinco determinantes directos presentados en la página 54, arrojaría un exceso de demanda de salud en un período determinado, que constituiría una presión por aumentar la disponibilidad hogareña o la disponibilidad ambiental de salud en los períodos siguientes. La otra posibilidad lógica, una producción en exceso de salud, no parece tener mayor significación empírica.

\section{Demanda familiar de SAlud}

Como se trata del resultado de una actividad productiva, entre los determinantes directos del nivel de salud deseado por el hogar incluyo las restricciones materiales a la apropiación hogareña de ése y de otros bienes $\longrightarrow$ sea, el poder adquisitivo y la estructura de costos de producción y precios-, y la restricción subjetiva representada por la estructura de gustos de los miembros del hogar. En vista de que la produc- 
ción de salud en el hogar significa generalmente producción conjunta de otros servicios de consumo (nutrición, vivienda, vestido, etc.), la demanda de salud estará relacionada positivamente con la demanda de los productos conjuntos de la salud. Los sustitutos de la salud son los servicios de consumo que debilitan la salud (como consumo de licores y tabaco), y aquellas actividades en las que la buena o mala salud no es un producto conjunto.

De acuerdo con las asociaciones empíricas observadas entre el poder adquisitivo hogareño y el precio relativo de muchas mercancías, por un lado, y la magnitud comprada de ese bien, por el orto, esperaríamos observar las siguientes relaciones en lo que a la magnitud deseada de salud se refiere:

1) A mayor poder adquisitivo hogareño, mayor magnitud de salud deseada. Esto conllevaría un desplazamiento dentro de la economía de tiempo del hogar desde las actividades sustitutas de la salud hacia las actividades complementarias de la salud. Por lo tanto, esperaríamos observar una mayor importancia relativa de los servicios de salud y de sus complementos en los presupuestos de hogares de mayor poder adquisitivo. Tal desplazamiento podría ser suavizado por diferencias entre hogares de diferentes ingresos en cuanto a su productividad en la producción de salud, tanto por la mayor escolaridad de los miembros de los hogares de ingreso mayor, como por las mejores técnicas usadas en esos hogares.

2) A mayor costo relativo de la salud o de los servicios de consumo complementarios de ella, menor demanda de servicios de salud. Es muy posible que hogares de mayor ingreso experimenten costos relativos más bajos que hogares de menor ingreso, puesto que los hogares más ricos, por motivo de su mayor escolaridad, son más eficientes y mejor informados, pudiendo así utilizar técnicas mejores, todo lo cual reduce su costo de producción de salud. Además, los hogares más ricos producen una gama de servicios de consumo (por ejemplo, viajes, vivienda, educación) que hogares más pobres no producen, o que éstos producen en mucho menor escala (12: S136). La disminución del costo relativo de la salud para los hogares más ricos, entonces, provendría también de que ellos pueden aprovechar, en mayor grado, la característica de la salud de ser un producto conjunto, y economías de escala en la producción hogareña de salud.

El efecto relativo de la estructura de gustos sobre la demanda hogareña de salud parecería ser mayor que el efecto relativo del poder adquisitivo hogareño y de la estructura de costos de producción y precios. En casi todas las culturas es muy preciada una vida larga y sana, con excepción de sectas especializadas, para quienes otros aspectos cobran más importancia. A reserva de modificar lo siguiente acorde con la evidencia concreta, supondré que la tasa de sustitución de salud por otros servicios de consumo es muy alta, reflejando una altísima preferen- 
cia en favor de la salud. Además, supondré que todas las personas, en un lugar y tiempo dados, desean por lo menos cierto nivel mínimo de salud. En el lenguaje de la microeconomía estática, esto implica una función de preferencia como la que aparece en la figura 1, donde $S_{\min }$ es el nivel mínimo de salud deseado por la persona en la fecha y lugar determinados. El origen representaría aquel nivel de salud debajo del cual sobreviene la muerte.

\section{Disponibilidad hogareña de salud}

Las actividades productivas realizadas en un hogar determinado pueden dividirse en: 1) producción de bienes materiales, tanto para el uso doméstico como para el intercambio; y 2) producción de servicios de consumo. Ambos grupos de actividades suelen tener a la salud del hogar en cuestión, así como a la de otros hogares, como un producto conjunto. El rendimiento unitario del trabajo en actividades hogareñas, en términos del producto conjunto salud, varía según el producto en cuestión, su escala de producción, la técnica y tecnología utilizadas, y las condiciones de trabajo en el hogar.

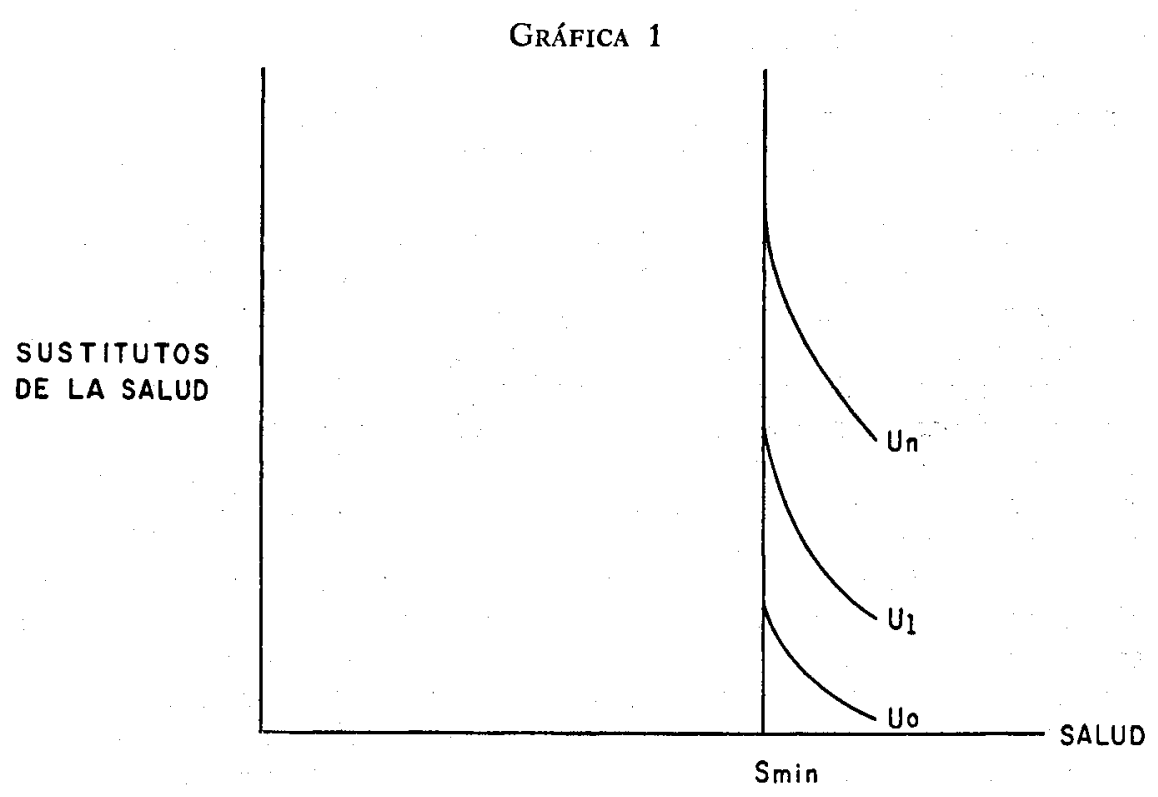

El desarrollo de las fuerzas productivas influye sobre la existencia de una demanda de mercancías producidas en hogares, al contribuir a la difusión del intercambio y al imprimir características concretas a la estructura de la demanda de mercancías. El desarrollo de las fuerzas productivas también influye sobre la existencia de una oferta hogareña 
de mercancías, al establecer la escala óptima de producción: si ésta rebasa la capacidad de producción del hogar, éste no podría sostenerse en la lucha competitiva con otras unidades productivas. La decisión de un hogar determinado de producir mercancías en su seno se verá afectada por la situación de clase del hogar, que le exige o no vender el producto de su trabajo para mantener y reproducirse. Las habilidades específicas del hogar condicionarán, en parte, qué mercancías específicas producirá. En cuanto a los servicios de consumo producidos en el hogar, su gama y escala de producción obedecerán a la existencia de sustitutos de esos servicios en el mercado, y al precio de los sustitutos, así como a las preferencias del hogar en cuestión. Por ejemplo, la invención de alimentos preparados que son relativamente baratos ha reducido el tiempo de trabajo hogareño dedicado a preparar comida (17: 119).

La cantidad de mercancías producidas en el hogar depende de la demanda de esas mercancías y de la capacidad productiva del hogar, la cual, a su vez, es función directa del poder adquisitivo del hogar, la estructura de costos y precios de los insumos, la teconología utilizada, la habilidad organizativa del hogar y las condiciones de trabajo en el hogar. Estas últimas podrían incluirse con la estructura de costos y precios, ya que el número de horas trabajadas por día, la frecuencia y duración de los períodos de descanso, la ventilación e iluminación del lugar de trabajo -que son algunas dimensiones de las condiciones de trabajo- se traducen de manera inmediata en costos de producción. Pero es mejor separar las condiciones de trabajo de los otros condicionantes directos de la producción hogareña, para así reconocer la posibilidad de una superexplotación del trabajo familiar.

La cantidad y calidad de servicios de consumo que puede producir un hogar también dependen de manera inmediata, del poder adquisitivo del hogar, de la estructura de costos y precios de los insumos, de la tecnología utilizada, de las condiciones de trabajo en el hogar y de la habilidad organizativa del hogar. La cantidad y calidad de los servicios de consumo deseados por el hogar son afectados por el poder adquisitivo del hogar, la estructura de costos y precios de los insumos, y las preferencias del hogar entre diferentes servicios de consumo. La interacción entre la demanda hogareña de servicios de consumo y la capacidad productiva hogareña de los mismos, establece la cantidad y calidad de servicios de consumo producidos en el hogar. Por ejemplo, los hogares de mayor ingreso tendrían mayor salud, porque podrían conseguir mayor cantidad y calidad de alimento, vivienda, ropa, escolaridad, atención médica, y así sucesivamente. Por otro lado, al disminuir el costo de algún insumo en la producción doméstica de salud, por ejemplo, de un antibiótico o del agua potable, aumentaría el uso del insumo en cuestión, con el consiguiente impacto positivo sobre la producción de salud en el hogar. La posibilidad antes mencionada (p. 56) de que los hogares de mayor ingreso tendrán costos relativos de salud más bajos que hogares de menor ingreso, reforzaría la diferencial tantas veces observada en la salud de hogares de diferente poder adquisitivo. 
No está de más repetir que la productividad, en términos de salud, no será igual para toda actividad productiva ni para todo nivel de esa actividad. Resalta aquí la importancia que revisten los conocimientos del hogar acerca del impacto que tienen las diferentes actividades sobre su salud, puesto que esos conocimientos pueden llevar al hogar a preferir una combinación de actividades productivas que maximizara la producción de salud en el hogar, de poder éste producir esa combinación óptima, atendiendo a las restricciones que representan, en primera instancia, su poder adquisitivo, la estructura de costos y precios y su capacidad organizativa y, en última instancia, su situación de clase. Es evidente que un hogar no escoge, necesariamente, entre todas las combinaciones de productos e insumos posibles, sino que escoge entre las que conoce. La información es el filtro que establece el conjunto de productos y técnicas que constituye el espacio de decisión del hogar. Hogares de mayor ingreso, por lo general, tienen mayor capacitación y experiencia en buscar y procesar información, lo que implica una mayor probabilidad de que tomen conocimiento de lo mejor que haya en tecnologías de salud en una fecha determinada. Además, los hogares de mayor ingreso están en condiciones de adquirir esa óptima tecnología de salud, que normalmente es más cara. Por el lado de los costos y precios relativos, una disminución en el costo de la información sobre las tecnologías aumentaría la eficiencia de la búsqueda para todos los hogares, aunque ese aumento sería mayor, en términos proporcionales, para hogares de menor ingreso. Esto conllevaría una difusión de las mejores tecnologías y un aumento generalizado de la salud en todos los hogares. Una reducción en el costo de uso de la tecnología más productiva tendría el mismo efecto.

\section{Disponibilidad ambiental de SAlud}

La contribución a la salud de un hogar dado, proveniente de las actividades de otros hogares y de la actividad extrahogareña, tendría tres componentes: las externalidades, en materia de salud, de las actividades productivas en otros hogares y de las actividades productivas extrahogareñas, y los efectos sobre la salud de los miembros del hogar en cuestión que nacen de su participación directa en las actividades productivas extrahogareñas.

En la magnitud de estos tres componentes inciden, de modo inmediato: las características específicas de los productos de la actividad hogareña y extrahogareña, la escala de producción y las técnicas utilizadas. En el caso de la transferencia de las externalidades negativas en materia de salud, tanto de las actividades hogareñas como de las extrahogareñas, participa también el poder político de la unidad productiva y su capacidad económica de absorber las externalidades negativas. Estos dos factores normalmente guardan una asociación positiva. En cuanto a las actividades extrahogareñas, habría que añadir las condiciones de trabajo como determinante directo. 
Las externalidades en materia de salud pueden ser positivas como pueden ser negativas: el signo que tome el saldo es cuestión concreta. Un hogar determinado podría, al adoptar nuevas prácticas - por ejemplo, hervir la leche-, disminuir el costo de información sobre esa nueva técnica para los otros hogares de su localidad. Un hogar que se vacuna durante una epidemia bloquea la difusión de ésta y contribuye así a la salud de otros hogares. Por el lado de las externalidades negativas, un hogar que arroja basura y efluentes a la calle pone en peligro la salud de los hogares cercanos. Anotaciones similares valen para las unidades extrahogareñas.

Las externalidades negativas representan costos privados que son transferidos a otras personas para ahorro del productor cuyas actividades los originan. Esta transferencia no siempre refleja el mayor poder político y la falta de solidaridad de la unidad productiva de origen. En muchos casos, el hogar original, por su bajo poder adquisitivo, materialmente no puede absorber tales costos, lo cual lleva a menudo a una segregación residencial -que es a la vez una segregación en niveles de salud- según el poder adquisitivo de los hogares, grabando en el espacio la desigualdad social que es el punto de partida de las diferencias en poder adquistivo de los hogares (4: cap. 2). Similarmente, habría que distinguir entre las unidades productivas extrahogareñas según su capacidad productiva, separando las medianas y pequeñas de las grandes. Las primeras gozan normalmente de márgenes de beneficio mucho más exiguos que los de las grandes unidades, y por consiguiente no están en condiciones de absorber los costos que transfieren al resto del mundo en la forma de externalidades negativas. Las unidades grandes, que sí podrían absorber esos costos con menor peligro para sus beneficios, no lo hacen, valiéndose de su poder político. De cualquier modo, tanto en uno como en otro caso, la continuada transferencia de costos hacia otras unidades extrahogareñas y hacia hogares, expresa la consolidación de control sobre el proceso de trabajo extrahogareño (4: cap. 5).

La diferencia fundamental entre las externalidades en materia de salud producidas en hogares $y$ en unidades extrahogareñas reside en que éstas, por razón de la tecnología vigente, tienen mucho más poder político que los hogares, pudiendo así transferir las externalidades negativas hacia el resto del mundo con más facilidad que la mayoria de los hogares (19: 171-2). Inclusive, es posible que las externalidades negativas en materia de salud de las actividades extrahogareñas sean mayores que las correspondientes externalidades negativas de las actividades hogareñas, especialmente ahora. Además, y a reserva de ver los resultados de estudios concretos, parecería que el saldo de las externalidades en materia de salud de las actividades extrahogareñas es negativo.

El impacto de las condiciones de trabajo en unidades extrahogareñas sobre la salud de sus trabajadores fue trazado de manera implacable por Marx y Engels (4; 10: cap. 8), y estudios posteriores han ratificado repetidamente los juicios de esos autores. En parte, las condiciones de trabajo reflejan la situación competitiva de la unidad extrahogareña en 
su lucha con otras unidades extrahogareñas. Además, esas condiciones reflejan la lucha entre quienes controlan el proceso de trabajo y los que carecen de poder de gestión, de tal modo que las condiciones de trabajo quizá podrían servir como indicador cuantitativo del grado de lucha de clase en cada rama de actividad. Salud de trabajadores y margen de ganancia suelen ser objetivos contradictorios en casi cualquier nivel que puedan cobrar ambas variables, como lo destaca un reciente artículo en una revista empresarial de circulación internacional, sobre la importante industria de cloruro de vinilo en los Estados Unidos (18).

\section{LA SALUD COMO INVERSIÓN}

Hasta este punto he considerado la salud como resultado de procesos de producción actual. Existe una corriente que considera la salud como inversión, o sea como un gasto actual encaminado a obtener o producir un flujo de poder adquisitivo en períodos futuros (véanse, por ejemplo, 6 y 14). Esta interpretación se aplicaría tanto a los gastos individuales como a los gastos sociales en salud.

En sentido estricto, es evidente que los gastos en salud representan una inversión, ya que mejor salud significa más horas laborables ( $y$, por consiguiente, más ingreso potencial), tanto por concepto de mayor sobrevivencia de la persona como por su menor morbilidad. Pero la interpretación de los gastos individuales en salud como una inversión del individuo en sí mismo, parece errónea, porque equivale a decir que la demanda de salud de una persona variará según el ingreso que espera obtener en las horas laborables adicionales que le proporcionaría la mejor salud. Tal elasticidad en la demanda de salud es algo por demostrar, y contradice el supuesto anteriormente enunciado, según el cual la demanda de salud, aunque no por necesidad es del todo inelástica, sí estaría por encima de la disponibilidad de salud. Más aún, el enfoque "inversionista" a nivel individual explicaría las diferenciales observadas en la salud de hogares de diferente poder adquisitivo (véase, por ejemplo, 2: 86-97), como resultado de diferenciales en la demanda de salud de esos hogares. Según esta óptica, los hogares de menor ingreso, quienes reciben un flujo de poder adquisitivo menor y más irregular que los hogares de mayor ingreso, reducirían su demanda de salud, ya que las expectativas económicas personales no justificarían tal inversión en sí mismos. La objeción a este planteamiento es clara: el bajo ingreso esperado de la persona, en vez de señalarle a éste que el beneficio marginal de gastos en su propia salud es bajo ( $y$, por lo tanto, que debiera reducir su demanda de salud), demuestra la baja capacidad de la persona de contribuir a su propia salud. La diferencia entre los dos enfoques radica en que uno hace énfasis en la selección por el individuo pobre de un bajo nivel deseado, mientras que el otro enfoque destaca el bajo ingreso personal como obstáculo a la producción personal de salud.

En contraste, sí cabría considerar los gastos estatales en salud como 
resultado parcial de la demanda de salud, considerada esta última como objeto de inversión pública. La interacción entre la demanda estatal de salud y de otros objetos de gasto público, por un lado, y el tamaño del erario, por el otro, establecería -en primera instancia- el monto del gasto público en salud, el cual podría impactar a todos los componentes de la disponibilidad de salud. Ese impacto está en aumento, como lo demuestran la creciente ingerencia estatal en determinar los costos de información y de uso de muchos bienes y servicios que afectan a la salud, así como la participación estatal en inventar bienes, servicios y técnicas relacionadas con la salud. También cabe notar el papel del estado en determinar quién absorbe las externalidades negativas, en materia de salud, de las actividades hogareñas y extrahogareñas. Al mismo tiempo, es fundamental recordar que la demanda estatal de salud no es una sencilla agregación de las demandas de una ciudadanía homogénea e igualitaria. Todo lo contrario. El estado, en tanto que instrumento de dominación de clase, refleja el grado de conflicto político en la sociedad. Por consiguiente, gastos estatales en salud -así como en educación y otros renglones del "bienestar social" - son, a la vez, resultados e indicadores del grado de conflicto existente en el país.

\section{RESUMEN}

1) El esquema aquí planteado especifica el conjunto de variables $y$ de relaciones causales que constituiría la determinación directa de la magnitud de salud personal, y permite trazar el impacto sobre la salud de variables como: ingreso por persona, condiciones habitacionales y sanitarias, nutrición, escolaridad, estructura ocupacional, gastos en salud pública, tecnología productiva y tecnología médica. Este esquema, con especificación de relaciones de retraso, podría entrar como módulo en un esquema teórico que integrara la determinación directa de la salud individual con los condicionantes indirectos de la misma, por ejemplo, la estructura de clases. Entre tanto, el presente esquema unifica hilos antes dispersos, al interpretar la salud como producto conjunto de muchos procesos de producción, entre los cuales se encuentran las actividades de consumo, tradicionalmente excluidas del ámbito de las actividades productivas. La magnitud de la salud producida dependería, entonces, del nivel de producción de los procesos en los que la salud es producto conjunto.

2) La mortalidad y la morbilidad hogareñas reflejan el nivel de salud del hogar, el cual provendría de la interacción entre: la demanda hogareña de salud, la disponibilidad hogareña de salud, la disponibilidad ambiental de salud, la virulencia de los agentes patógenos y de los vectores de enfermedad, y la composición genética de la población. Todas estas variables son referidas al mismo período de tiempo, aunque seguramente hay relaciones de rezago, las cuales habría que especificar al utilizar el presente esquema en la investigación concreta. 
3) Parece razonable suponer que siempre ha existido un exceso de demanda de salud, y que los efectos sobre la salud hogareña provenientes de la virulencia de los agentes pátogenos y de los vectores de enfermedad, así como de la composición genética de la población misma, se producen con gran rezago. En tales condiciones, sería la disponibilidad de salud la que determinaría, de manera directa, la magnitud de la salud observada en los hogares.

4) La disponibilidad de salud resulta de una amplia gama de actividades productivas clasificables según quien detente el poder de gestión en cada una de ellas. A la disponibilidad de salud contribuyen la disponibilidad hogareña de salud y la disponibilidad ambiental de salud. La disponibilidad hogareña representa la contribución, en materia de salud, de aquellas actividades hogareñas de producción y consumo en las que la buena o mala salud es un producto conjunto. La disponibilidad ambiental de salud tiene tres componentes: $a$ ) las externalidades, en materia de salud, de las actividades hogareñas de producción y consumo; $b$ ) las externalidades, en materia de salud, de las actividades extrahogareñas de producción y consumo; y c) los efectos de las actividades extrahogareñas sobre la salud de las personas que participan en ellas.

5) Un enfoque paralelo, que considera la salud como una inversión, destaca la demanda de salud como determinante de la magnitud observada de salud personal. Este enfoque presupone una elasticidad de la demanda individual de salud que parece, a priori, equivocadamente alta. Sin embargo, es posible que la demanda estatal de salud - considerada ésta como demanda estatal de un tipo de inversión- sea significativamente positiva e influya sobre la contribución del estado a la disponibilidad de salud. En este caso, es importante reconocer que la demanda estatal de salud no es necesariamente una agregación equitativa de las demandas individuales de salud.

\section{BIBLIOGRAFİA}

1. G. Becker, "A Theory of the Allocation of Time", Economic Journal, 75 (299), septiembre de 1965: 493-517.

2. Hugo Behm Rosas, Mortalidad infantil y nivel de vida. Santiago de Chile, Universidad de Chile, 1962.

3. L. L. Cavalli-Sforza, "The Genetics of Human Populations", Scientific American, 231 (3), septiembre de 1974: 81-89.

4. F. Engels, La situación de la clase obrera en Inglaterra, Buenos Aires, Diaspora, 1974.

5. R. Ferber, "Consumer Economics. A Survey", Journal of Economic Literature, 11 (4), diciembre de 1973: 1303-42.

6. M. Grossman, "On the Concept of Health Capital and the Demand for Health", Journal of Political Economy, 80, marzo/abril de 1972: 223-55.

7. K. Lancaster, "A New Approach to Consumer Theory", Journal of Political Economy, 74, abril de 1966: 132-157.

8. K. Lancaster, Consumer Demand: A New Approach. Nueva York, Columbia University Press, 1971. 
9. K. Marx, Elementos fundamentales para la crítica de la economía política (borrador) 1857-1858. Vol. 1. México, Siglo XXI, 1971.

10. K. Marx, El Capital, Vol. I. Traducción de Wenceslao Roces, 2a edición en español. México, Fondo de Cultura Económica, 1959.

11. K. Marx, y F. Engels, "Feuerbach. Oposición entre las concepciones materialistas e idealistas", en K. Marx y F. Engels, Obras escogidas, en tres tomos, Tomo I. Moscú, Editorial Progreso. 1973: 11-81.

12. R. T. Michael, "Education and the Derived Demand for Children", Journal of Political Economy, 81 (2, parte II), marzo/abril de 1973: S128-64.

13. I. Mincer, "Market Prices, Opportunity Costs, and Income Effects", en C. Christ et al. (Comp.), Measurement in Economics: Studies in Mathematical Economics and Econometrics in Memory of Yehuda Grunfeld. Stanford, 1963.

14. S. J. Mushkin, "Health as an Investment", Journal of Political Economy, 70 (5, parte 2), octubre de 1962: 129-57.

15. T. W. Schultz, (Comp.), "New Economic Approaches to Fertility. Proceedings of a Conference, June 8-9, 1972", Journal of Political Economy, 81 (2, parte II), marzo/abril de 1973 .

16. T. W. Schultz, (Comp.) "Marriage, Family Human Capital, and Fertility. Proceedings of a Conference, June 4-5, 1973", Journal of Political Economy, 82 (2, parte II), marzo/abril de 1974.

17. J. Vanek, "Time Spent in Housework", Scientific American, 231, noviembre de 1974: 116-20.

18. P. H. Weaver, "On the Horns of the Vinyl Chloride Dilemma", Fortune, 90 (4), octubre de 1974.

19. E. R. Weiss-Altaner, "Explosión demográfica y tensión económica", DemograFíA Y ECONOMía, 7 (2), 1973: 164-174. 